\title{
Isolation of Lineage Specific Nuclei Based on Distinct Endoreduplication Levels and Tissue-Specific Markers to Study Chromatin Accessibility Landscapes
}

\author{
Ezgi Süheyla Karaaslan ${ }^{1}\left(\mathbb{D}\right.$, Natalie Faiß ${ }^{1}$, Chang Liu ${ }^{1,2, *(1)}$ and Kenneth Wayne Berendzen ${ }^{1, *(1)}$ \\ 1 Center for Plant Molecular Biology (ZMBP), University of Tübingen, Auf der Morgenstelle 32, \\ 72076 Tübingen, Germany; ezgi.dogan@zmbp.uni-tuebingen.de (E.S.K.); \\ natalie.faiss@zmbp.uni-tuebingen.de (N.F.) \\ 2 Institute of Biology, University of Hohenheim, Garbenstrasse 30, 70599 Stuttgart, Germany \\ * Correspondence: chang.liu@uni-hohenheim.de (C.L.); kenneth.berendzen@zmbp.uni-tuebingen.de (K.W.B.); \\ Tel.: +49-711-459-22180 (C.L.); +49-7071-78721 (K.W.B.)
}

Received: 30 September 2020; Accepted: 1 November 2020; Published: 3 November 2020

check for updates

\begin{abstract}
The capacity for achieving immense specificity and resolution in science increases day to day. Fluorescence-activated nuclear sorting (FANS) offers this great precision, enabling one to count and separate distinct types of nuclei from specific cells of heterogeneous mixtures. We developed a workflow to collect nuclei from Arabidopsis thaliana by FANS according to cell lineage and endopolyploidy level with high efficiency. We sorted GFP-labeled nuclei with different ploidy levels from the epidermal tissue layer of three-day, dark-grown hypocotyls followed by a shift to light for one day and compared them to plants left in the dark. We then accessed early chromatin accessibility patterns associated with skotomorphogenesis and photomorphogenesis by the assay for transposase-accessible chromatin using sequencing (ATAC-seq) within primarily stomatal 2C and fully endoreduplicated $16 \mathrm{C}$ nuclei. Our quantitative analysis shows that dark- and light-treated samples in $2 \mathrm{C}$ nuclei do not exhibit any different chromatin accessibility landscapes, whereas changes in $16 \mathrm{C}$ can be linked to transcriptional changes involved in light response.
\end{abstract}

Keywords: FANS; endopolyploidy; skotomorphogenesis; photomorphogenesis; ATAC-seq; hypocotyl; hromatin accessibility

\section{Introduction}

In plant development, light acts as a critical environmental modulator at nearly every stage. In order to reach the surface, seeds germinating under the soil direct resources to elongate the hypocotyl by a developmental program called skotomorphogenesis. Skotomorphogenesis is crucial for plant survival and is characterized not only by rapid hypocotyl growth, but also by a closed apical hook to protect the apical meristem from mechanical damage. On the contrary, seedlings exposed to light undergo photomorphogenesis, an antagonistic developmental progress that is characterized by a short hypocotyl and open cotyledons [1]. Such a delicate developmental balance is tightly regulated at the transcriptional level by various transcription factors cascades [2]. In Arabidopsis, PHYTOCHROME INTERACTING FACTORS (PIFs) are well-characterized central regulators of skotomorphogenesis. PIFs are basic helix-loop-helix transcription factors (TFs) which constitutively suppress photomorphogenesis in young seedlings by directly binding to the G-Box elements in the promoters of several target genes [3-7]. PIF1, PIF3, PIF4 and PIF5 play overlapping roles in promoting dark state development: in the quadruple mutant (pifq), skotomorphogenesis is interrupted and results in a shorter hypocotyl and open cotyledons [8-10]. Upon initial exposure to light, PIFs are rapidly 
phosphorylated by photo-activated phytochromes and subsequently degraded by the $26 \mathrm{~S}$ proteasome, initiating photomorphogenesis [4,11-13]. Another transcription factor, ELONGATED HYPOCOTYL 5 (HY5), has a major role in regulating photomorphogenesis [14]. This bZIP TF interacts with light response genes by preferentially binding to G-Box motifs, but also LREs Z-CA- and CG-hybrid boxes elements at their promoter regions [3,15]. Several immunoprecipitation and transcriptome analyses have shown that HY5 regulates thousands of genes in the Arabidopsis genome [15-17]. There is emerging evidence that not only transcription factors, but also histone modifiers, are involved in the regulation of dark-to-light transition of plants. Several studies have shown opposing regulatory activities of histone acetyltransferases (HATs) and deacetylases (HDACs) following light perception [18-20]. It is crucial to investigate the differences in chromatin accessibility patterns throughout the hypocotyl as chromatin states and histone modifications at various plant developmental stages in response to environmental cues are gaining importance [21,22].

Endoreduplication, also called somatic polyploidization or endopolyploidy, is coupled to development in many plant species [23]. Endoreduplication occurs in all cell types except gametes, guard cells, meristematic cells and floral organs [24]. Increased DNA amount as a result of endoreduplication events is closely associated with cell growth in many organisms [25-27] and in dicotyledonous plants, endoreduplication in hypocotyl tissue is an essential part of skotomorphogenesis. The Arabidopsis hypocotyl is composed of only 20 cell files; several endoreduplication cycles in the hypocotyl tissue provide cell expansion and therefore tissue expansion [2,28]. It is known that one extra endoreduplication cycle is observed in dark-grown hypocotyls compared to light-grown hypocotyls [28], and this is inhibited via various photoreceptors during photomorphogenesis [2,28]. The phytohormones gibberellin, auxin and brassinolide clearly promote hypocotyl elongation in dark-grown plants $[2,4,29]$, whereas abscisic acid inhibits hypocotyl elongation when gibberellic acid and auxin concentrations are low [30]. Although the exact mechanism of how ploidy levels regulate cell growth remains elusive [31], it has been proposed that increased transcriptional and metabolic activity due to the multiplication of genetic material contributes to cell growth [32-37].

Gene expression is closely connected to chromatin structure. Currently, it is unclear to what extent chromatin accessibility of individual genes varies during the developmental transition from skotomorphogenesis to photomorphogenesis is coupled with changes in endoreduplication activities. Flow cytometry can be used to purify nuclei from tissue homogenates [38], making it possible to harvest cell-type-specific nuclei from plant tissues using nuclear-targeted fluorescent proteins, as demonstrated for studying endoreduplication within specific cell types in the root [39] or exploring the transcriptome of genes selectively expressed in the phloem [40]. Studying the changes in chromatin accessibility that occur upon light exposure may provide us with a better view on how light initiates different regulatory mechanisms that are dependent on chromatin structure changes. In this study, we performed the assay for transposase-accessible chromatin using sequencing (ATAC-seq), a widely used high-throughput method to detect open chromatin regions, to assess chromatin accessibility in a tissue-, lineage- and endopolyploidy-specific manner. Such an assay on this particular tissue and cell type has never been done before.

\section{Results}

\subsection{Isolation of Lineage-Specific Nuclei Based on Endopolyploidy Levels and a Tissue-Specific Marker for ATAC-seq}

Since the association between skotomorphogenesis and cellular endoreduplication has been demonstrated in epidermal cell layers of the hypocotyl [28,41], we used an epidermal tissue-specific marker $p A t M L 1:: N L S: G F P$ line to obtain GFP-tagged epidermal nuclei from plants growing under different light conditions. Along with specifically harvesting GFP-positive nuclei by fluorescence activated nuclei sorting (FANS) [42], we further separated them according to their endopolyploidy levels and selected 2C and 16C to perform ATAC-seq (Figure 1). Three-day-old Arabidopsis seedlings were grown in complete darkness, then transferred into the light for one day in order to collect 
nuclei for light-treated samples. Seedlings were kept in the dark for an additional day to collect nuclei from dark-treated samples (Figure 1A). This 1 day exposure window allows plants to transition from skotomorphogenesis to photomorphogenesis exactly when the hypocotyl grows the most [28] (Figure 1B). Several morphological differences were observed after one day of change: upon light exposure, the cotyledons opened and hypocotyls ceased their rapid elongation, whereas the dark-grown plants practically doubled in hypocotyl length compared to the light-grown plants (Figure 1B). This is consistent with what has been previously observed [28].

Chemical fixation preserves biological materials for downstream processes $[43,44]$. To get the best ATAC-seq results, we fixed the hypocotyls prior to FANS using $4 \%$ formaldehyde (FA), as we found that this concentration had superior nuclear GFP signals compared to $1 \%$, yielding clearly discernible ATAC-seq peaks useful for downstream analysis (Figure S1). After fixation, the hypocotyl was carefully excised by removing the cotyledons and roots and stored at $-8{ }^{\circ} \mathrm{C}$. To extract nuclei, the tissue was chopped with a razor blade in buffer for minimal structural disruption and filtered twice through $40 \mu \mathrm{m}$ nylon mesh. Nuclei were stained with DAPI and analyzed by flow cytometry before FANS (Figure 1C,D). In both skotomorphogenic and photomorphogenic plants, multiple nuclear DAPI (Figure 1D-G) and GFP (Figure 1E-G) populations were observed due to endoreduplication events occurring within the hypocotyl tissue. The most extreme ploidy levels were sorted, 2C and 16C, based on their DAPI and GFP signal (Figure 1E,F). After obtaining an adequate number of nuclei by FANS, we proceeded with the Tn5 integration step of the ATAC-seq protocol.

Most of the GFP expressing nuclei were at the $8 \mathrm{C}$ and 16C stage (Figure 1F,G). According to the counts extracted from the FANS sort data, our analysis shows that epidermal layer cells are the main contributors of high endopolyploidy nuclei in the hypocotyl comprising $\sim 80 \%$ and $\sim 66 \%$ of all 16C nuclei in the dark- and light-treated samples, respectively (Figure $1 \mathrm{H}$ ). This corroborates early microscopy-based observations that the epidermal and cortical cells contain the highest DNA levels [41]. The shift from dark to light also correlated with a reduction in the percentage of $8 \mathrm{C}$ and $16 \mathrm{C}$ endoreduplicated nuclei (Figure $1 \mathrm{H}$ ), indicating that the switch from skoto- to photomorphogenesis leads to a halt in the endoreduplication process. These results indicate that we have collected material for ATAC-seq from the major hypocotyl cell layer involving endoreduplicated nuclei. 
A

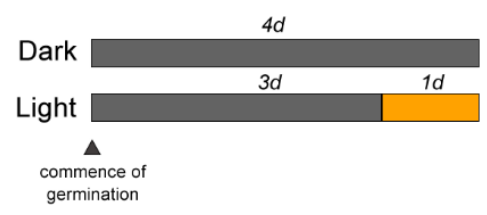

B

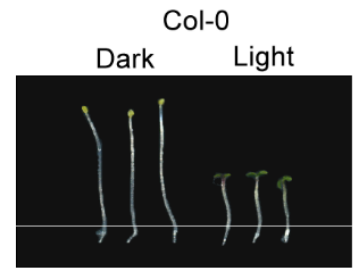

C
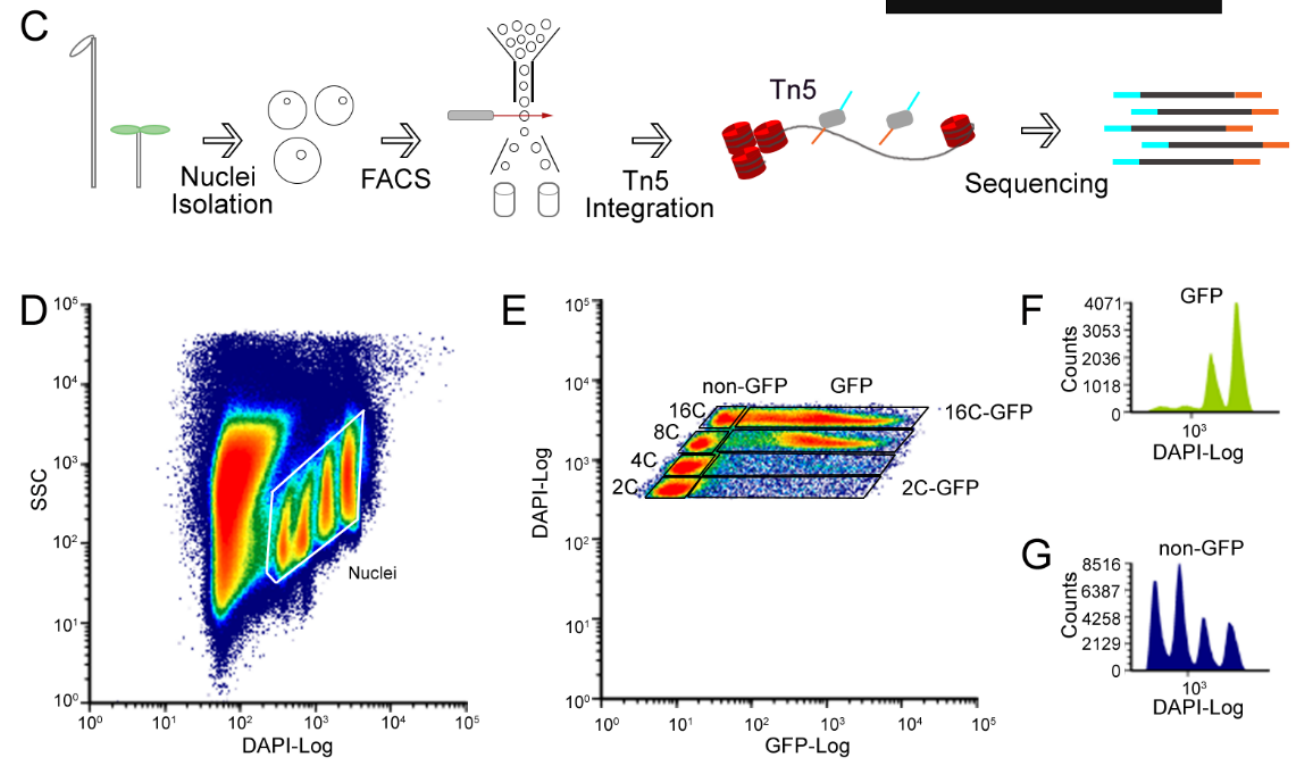

$\mathrm{H}$

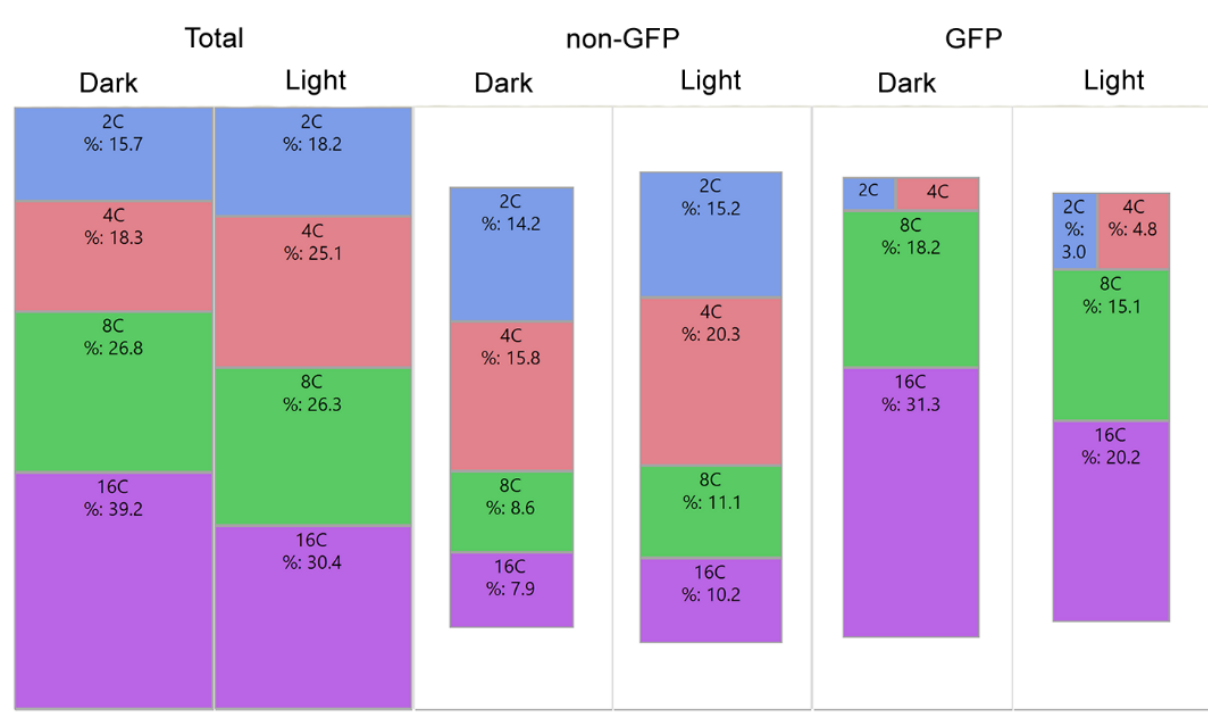

Figure 1. Endopolyploidy levels increase in dark-grown hypocotyls specifically in the epidermal layer. (A) Experimental design for light and dark-grown hypocotyls. (B) Representative images of light- and dark-grown Col-0 hypocotyls. (C) Workflow of fluorescence activated nuclei sorting (FANS) combined with the assay for transposase-accessible chromatin using sequencing (ATAC-seq). (D) Bivariate density histogram of the initial gate used to separate nuclei from major debris. See methods for further channel clean up. (E) Bivariate density histogram of the final gates used for sorting after clean-up for all endoploidy regions. (F) DAPI signal for all nuclei expressing the GFP marker. (G) DAPI signal for all nuclei expressing the not expressing the GFP marker. $(\mathbf{H})$ TreeMap view of the percentage of nuclei for each endoreduplication level for a typical sort. The size of the rectangles is proportional to the sample size. 


\subsection{ATAC-seq Revealed Accessible Chromatin Patterns in Dark and Light Grown Hypocotyls}

After performing ATAC-seq for light- and dark-treated hypocotyls in $2 \mathrm{C}$ and $16 \mathrm{C}$ nuclei, we identified roughly $\sim 38,000$ peaks across all samples. Our initial analysis of the ATAC-seq data revealed that the global chromatin accessibility landscape throughout the Arabidopsis genome is very similar among the different samples (Figure 2A). As several transcription factors are involved in the skoto- to photomorphogenesis transition, we analyzed local changes in chromatin accessibility upon light exposure around TF binding sites. Considering the important regulatory roles of the PIF transcription factor family and HY5 during this transition, we used previously published ChIP-seq data to analyze potential differences in chromatin accessibility around PIFs and HY5 binding sites. We selected PIF ChIP-seq data from Pfeiffer et al. [45] and HY5 ChIP-seq data from Burko and colleagues [46]. Our ATAC-seq analysis revealed no changes in local chromatin accessibility around PIF1, PIF3, PIF4, PIF5 and HY5-binding DNA regions in neither 2C nor 16C nuclei (Figure 2 and Figure S2). As not only PIF targets, but also PIF genes are regulated at the transcriptional level $[5,47]$, we inspected PIF1 and PIF3 for differences in chromatin accessibility around their gene bodies and promoters. Like the global pattern, both PIF1 and PIF3 displayed similar chromatin accessibility in dark and light hypocotyl in both $2 \mathrm{C}$ and $16 \mathrm{C}$ nuclei (Figure S3).
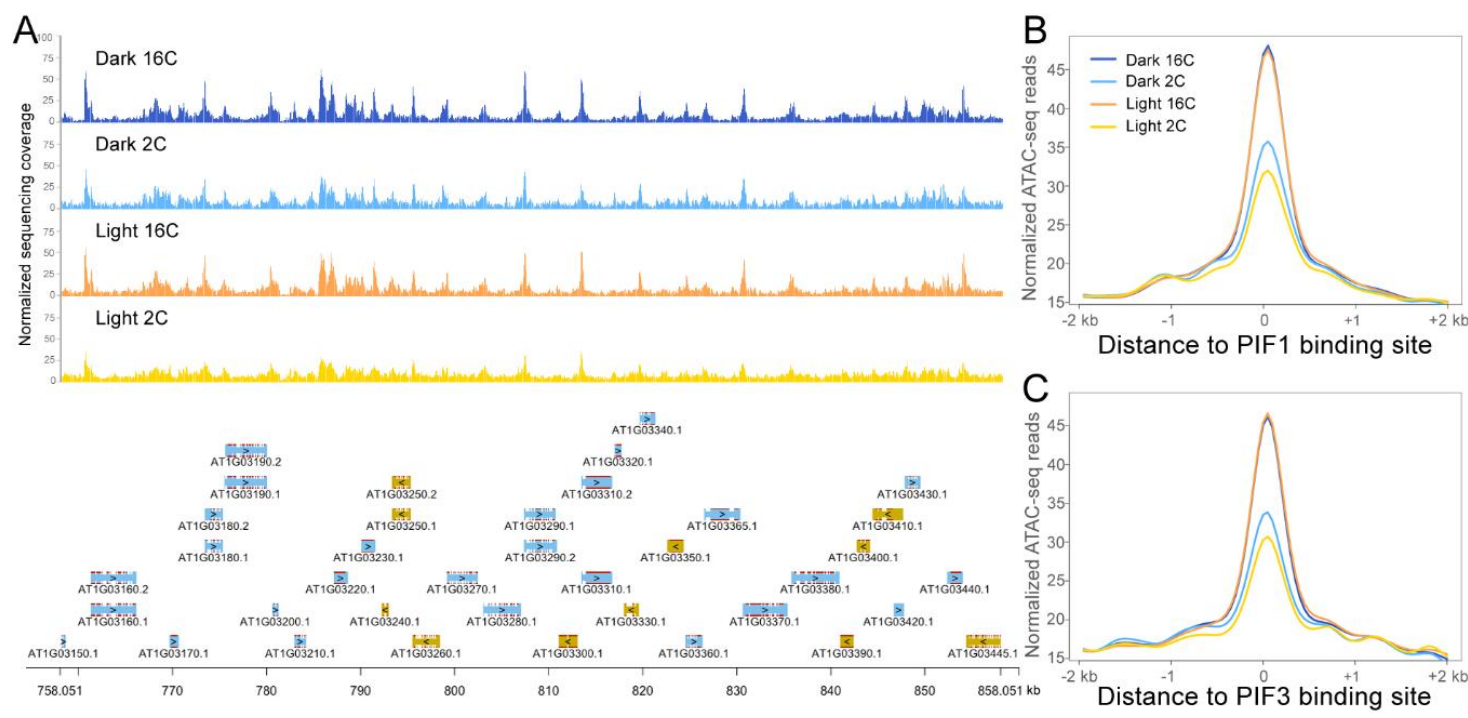

Figure 2. Chromatin accessibility patterns of $2 \mathrm{C}$ and $16 \mathrm{C}$ nuclei from dark and light treated hypocotyls. (A) Representative ATAC-seq peaks of different samples from a $100 \mathrm{~kb}$ genomic region of Chr1. In the panel below, genes indicated by blue are on the "Watson" strand and yellow ones are on the "Crick" strand. ATAC-seq read density is plotted with $50 \mathrm{bp}$ windows. Normalized ATAC-seq signals around PIF1 (B) and PIF3 (C) transcription factor (TF) binding sites.

Thereafter, we performed quantitative analysis to identify differentially accessible chromatin regions from light and dark samples in 2C and 16C nuclei (see the "Materials and Methods" section). When we compared light- and dark-treated $2 \mathrm{C}$ nuclei, our quantitative analysis revealed that only one region was more accessible in light-treated samples and no region was more accessible in dark-treated samples. This means light- and dark-treated $2 \mathrm{C}$ nuclei have identical open chromatin landscapes. Darkand light-treated 16C nuclei, on the other hand, had a significant number of differential ATAC-seq peaks. Comparing light- and dark-treated $16 \mathrm{C}$ nuclei revealed, on one hand, that 70 regions that were more accessible in light samples; we will refer to them from now on as light accessible regions (LARs). On the other hand, 20 regions were more accessible in 16C dark samples, which we refer to from now on as dark accessible regions (DARs). We then determined which genes are overlapping with LARs and DARs. We considered a gene as a 'differentially accessible gene' if the genomic region from $1000 \mathrm{bp}$ upstream of the annotated transcription start site (TSS) to its transcription termination 
site (TTS) is overlapping with one or more LAR or DAR. Only one gene, AT3G44970, encoding a cytochrome P450 superfamily protein, was more accessible in $2 \mathrm{C}$ light samples (Table S1). This gene is expressed specifically in the hypocotyl according to a Kelpikova Atlas eFP Browser analysis (Figure S4). Upon light exposure in 16C nuclei, 56 genes from 54 out of 70 LARs were more accessible in the light-treated samples and 11 genes from 11 out of 20 DARs were more accessible in dark-treated samples (Table S1). Therefore, we focused only on 16C nuclei for further analysis of differentially accessible regions. Examples of two genes with LARs are shown in Figure 3A,B.

A

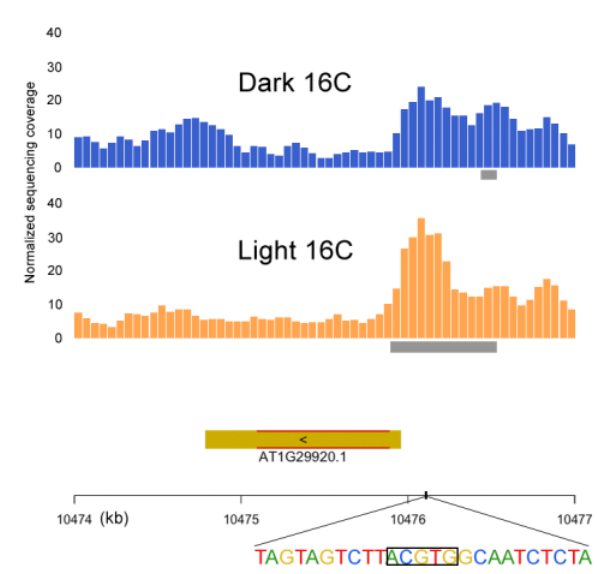

C photosynthesis, light harvesting

$$
\begin{array}{r}
\text { photosynthesis } \\
\text { response to far red light } \\
\text { response to red light } \\
\text { photosynthesis, light reaction } \\
\text { response to blue light } \\
\text { response to light stimulus } \\
\text { response to red or far red light } \\
\text { response to radiation } \\
\text { generation of precursor } \\
\text { metabolites and energy } \\
\text { organic substance metabolic process } \\
\text { carbon fixation }
\end{array}
$$

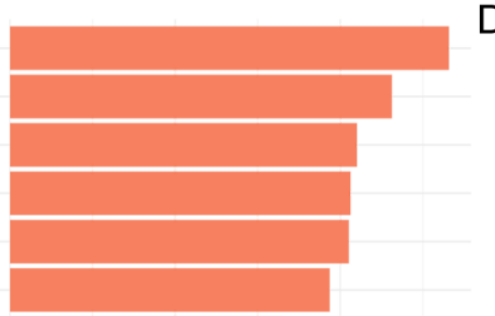

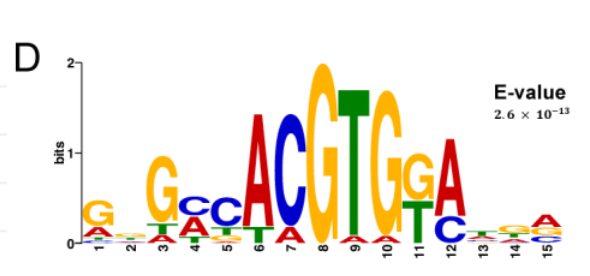

B

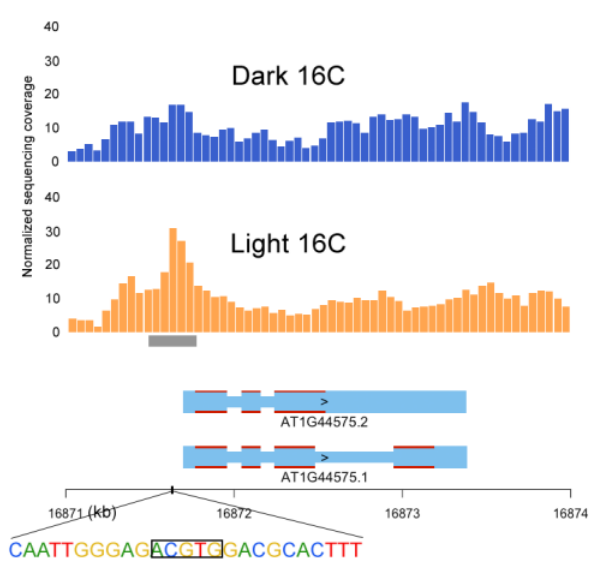

Figure 3. Analysis of differentially accessible genes and regions of dark and light hypocotyls. (A) Differential light accessible regions (LAR) ATAC-seq peaks around the promoter of CHLOROPHYLL A/B-BINDING PROTEIN 2 (CAB2) and (B) NONPHOTOCHEMICAL QUENCHING 4 (NPQ4). Both genes are on Chr1. Grey bars indicate ATAC-seq peaks annotated by MACS2. (C) Gene Ontology (GO) analysis of the 56 genes that are more accessible in light treated hypocotyl in $16 \mathrm{C}$ nuclei. (D) Sequence Logo of the recovered G-Box motif from the Multiple Em for Motif Elicitation (MEME) motif analysis of all LARs.

We next performed Gene Ontology (GO) analysis on the two groups of $16 \mathrm{C}$ genes. The 11 genes that are more accessible in dark-treated samples did not show any GO enrichment ( $p$-value cutoff set as 0.05). In contrast, $\mathrm{GO}$ analysis for the 56 genes more accessible upon light exposure revealed that the set is enriched for photosynthesis, response to light and metabolite processing (Figure 3C; Table S2). The LAR genes appear to be quite diverse. Nevertheless, 14 of the 56 are core photosynthesis genes, there are several transcription factors, two genes involved in ABA signaling, and a few genes that can 
be linked to chromatin regulation. Five genes are also known to produce mobile signals at the RNA level [48]. The genes harboring DARs are also fairly diverse, yet three also produce growth factors or mobile signals (Table S3).

Furthermore, in order to determine candidate transcription factors located in differentially accessible regions, we performed Multiple Em for Motif Elicitation (MEME) motif analysis [49] on LAR and DARs. Only two significant motifs were returned for LARs and none for DARs (Figure S5). The strongest hit $\left(p=2.6 \times 10^{-13}\right)$ was the motif "ACGTG" (Figure 3D), which is strongly bound by HY5 [46] and indirectly by PIFs [50]. The tetra-nucleotide "ACGT" is the typical core recognition sequence for plant bZIP transcription factors [51-53] and the "ACGTGK" motif, also called ABA-RESPONSIVE ELEMENT (ABRE), is found in promoters of many abscisic acid (ABA)-inducible genes, conferring ABA responsiveness [52,54].

\subsection{Analysis of Identified Differentially Accessible Genes for Regulation by Light by Mining Publicly Available Expression Data}

We decided to investigate if there is any evidence that the gene sets we obtained are regulated by light at the transcriptional level. For 46/56 of the LAR genes present in the AtGenExpress Light Treatments dataset, 17 of 46 of them are actually up-regulated by red, far-red, blue and/or UV-light in wild-type Col-0 seedlings. Three of the 9/11 DAR genes on the Affymetrix chip are down-regulated by different light qualities (Figure 4; Table S4). Not a single gene was found to be two-fold or more inversely regulated in either dataset. Thus, we see that the ATAC-seq dataset faithfully captured light-regulated regions whose chromatin environment can also be mirrored in transcriptional changes.

In addition, we also compared the LAR and DAR genes with a list of genes specifically identified as light-regulated in the hypocotyl and cotyledons in Arabidopsis (Col-0) seedlings from Sun et al. [55]. In this RNA-seq analysis, plants were kept in continuous darkness for four days and then transferred to white light for 1 or $6 \mathrm{~h}$, whereafter cotyledons and hypocotyls were dissected. Approximately $67 \%$ (38/56) of all the LARs and 63\% (7/11) of the DARs are found in their complete dataset. Of the $67 \%$ LAR genes that overlap, only $7 / 38$ are labeled as cotyledon-specific, meaning that $55 \%$ of our LAR genes are regulated specifically in the hypocotyl according to Sun et al. Similarly, applying the same analysis, we find $4 / 11$ (36\%) of the DAR genes specifically regulated in the hypocotyl (Table S5).

If we consider only the LAR genes and compare them only to those genes regulated after $6 \mathrm{~h}$ of light treatment from hypocotyl tissue from the Sun et al. experiment, around 50\% (28/56) of the more accessible LAR genes are actually upregulated in the RNA-seq dataset, whereas 36\% (4/11) of DAR genes are down-regulated (Table S5). Thus, the changes occurring in chromatin accessibility appear to result in transcriptional reprogramming, as the genes that are falling into LARs or DARs are also up- or down-regulated in the RNA-seq as well. This result is consistent with the AtGenExpress light experiment analysis shown above (Figure 4). We also tested the chromatin accessibility of the total list of 3400 upregulated genes in this particular RNA-seq dataset in our ATAC-seq dataset. These genes, however, displayed similar levels of chromatin accessibility in all samples, which is quite open in the region at, and directly upstream of, the TSS (Figure S6). In summary, we observe very few differential chromatin changes in the $2 \mathrm{C}$ and $16 \mathrm{C}$ of epidermal cells. Nevertheless, the genes we identified as having light-dependent chromatin accessibility changes in the $16 \mathrm{C}$ are paralleled in light-dependent transcriptional changes, meaning that transcriptional reprogramming can directly be associated with changes in the chromatin accessibility for at least the $16 \mathrm{C}$ cell lineage. 

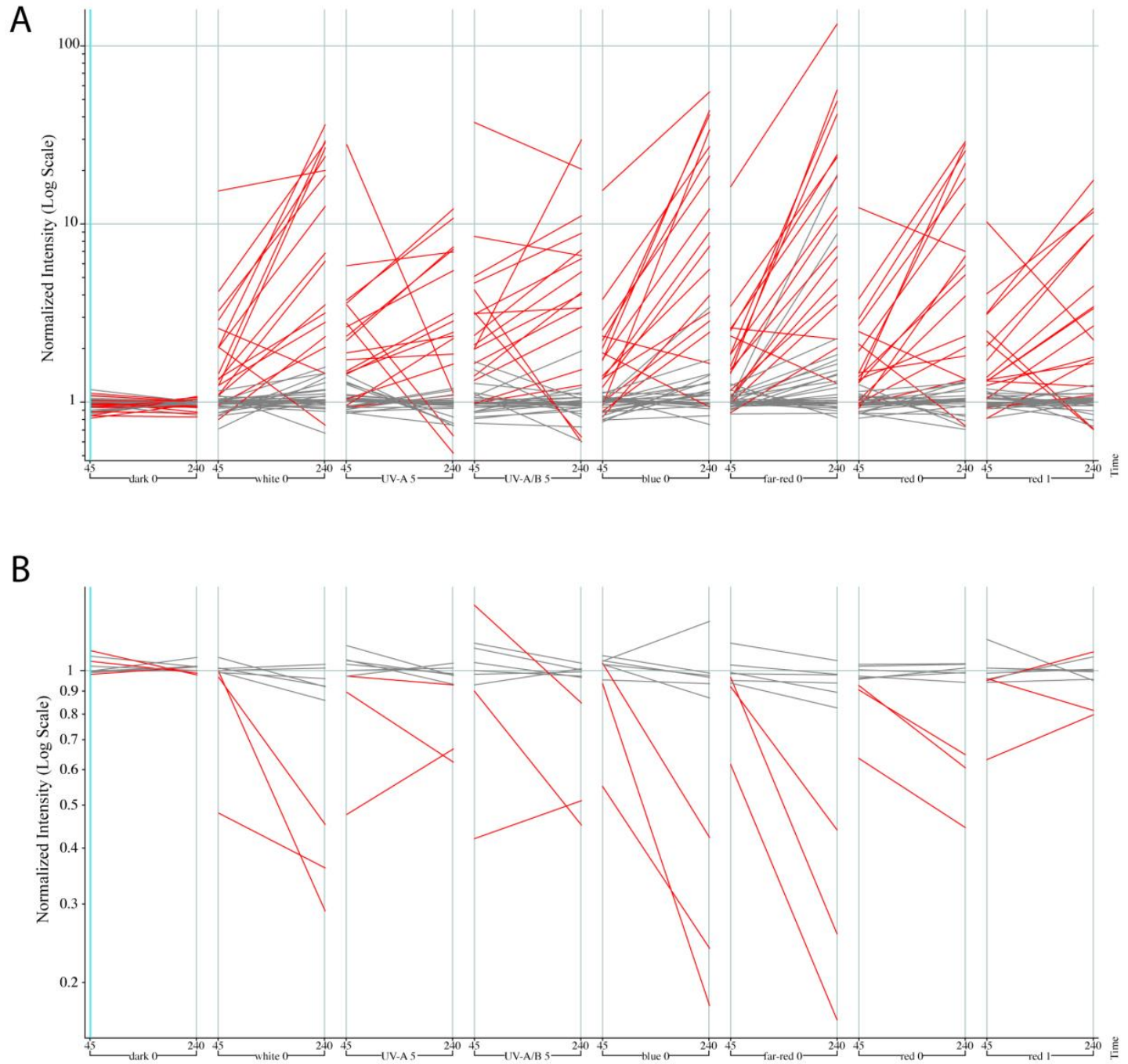

Figure 4. Regulation by light for LAR and DAR genes in the AtGenExpress: "Light Treatments" experiment. (A) The 46 out of 56 LAR genes found in the dataset, with seventeen two-fold up-regulated in white light, highlighted in red. (B) The 9 out of 11 DAR genes found in the dataset, with three two-fold down-regulated in white light, highlighted in red.

\section{Discussion}

Light is one of the most important abiotic factors regulating plant growth and development. In this study, we have established a nuclear ATAC-seq workflow that allows one to work on specific cell pools from individual tissue layers. In particular, FANS enabled us to distinguish, sort and analyze 2C and 16C endoreduplicated epidermal cells from the hypocotyl. In our analysis, we identified $\sim 38,000$ peaks by ATAC-seq, which could be mapped to 90 differentially accessible dark-to-light regions, corresponding to 67 differentially accessible genes among $16 \mathrm{C}$ nuclei and one in $2 \mathrm{C}$ nuclei. Overall, our ATAC-seq analysis reveals that global chromatin accessibility patterns among dark and light treated epidermal hypocotyl 2C and 16C nuclei did not show dramatic changes after one day of white light exposure.

It has been demonstrated that light-responsive cis-elements are localized in close proximity to the TSS [16]. PIFs and HY5 regulate $\sim 20 \%$ of the genome, controlling hormonal pathways and themselves being controlled by phytochromes to modulate photomorphogenesis [2]. PIFs accumulate in darkness, are essential for skotomorphogenesis, and are the major suppressors of photomorphogenesis, promoting hypocotyl elongation and inhibiting chloroplast differentiation (reviewed in [56]), whereas HY5 in the light is the major repressor of skotomorphic PIF function [5]. PIFs bind to G-boxes, but also 
E-, PBE-boxes and G-box coupling elements [3] and similarly, HY5 binds to G-boxes, but also LREs Z-CA- and CG-hybrid boxes elements [3,51]. The HY5 binding motif stood out as a main TF binding motif in LARs (Figure 3D and Figure S5), but could be a target of both TFs. Loss of HY5 leads to a long hypocotyl under red, far-red, blue and white light [57]. Many of our 67 genes are regulated by one or more of these light qualities (Figure 4), suggestive of a direction connection to HY5. The physiological response to light in the hypocotyl was abrupt and evident after $24 \mathrm{~h}$ (Figure 1B); globally, however, the chromatin around TSS regions was already open such that the fate for both cell types appears to be predetermined. Using ChIP-seq against native HY5, Zhang et al. 2011 [15] reported that while it bound to $\sim 11,000$ genes under their experimental conditions, only a tenth of those had altered gene expression, and $60 \%$ of the HY5 bound regions were bound irrespective of the light/dark conditions. This could also be the case here, where the chromatin environment is already set but waiting for particular sets of controlling factors to initiate developmental programs. In this regard, it is not so surprising that we uncover only a few changes in very specific cell types then. It has been shown, however, that light signaling triggers chromatin rearrangements in different developmental stages [21]. This indicates that most likely, major changes in chromatin accessibility, and likely transcriptional regulation, occur either at the other ploidy levels in the epidermis, and/or in other cell types from the hypocotyl. Alternatively, maybe longer-term changes are recorded in the chromatin, but are not part of the early photomorphogenic response, at least in these particular cell types. Nevertheless, for those differential accessibility regions detected in $16 \mathrm{C}$ nuclei, we clearly detected genes that respond to light to mediate photomorphogenesis and respond to light at the chromatin level.

With regard to $2 \mathrm{C}$ nuclei, almost no other cell divisions in the hypocotyl occur after seed imbibition [28,29], such that the very few to no cell divisions that do occur, are only in the cortex or epidermal hypocotyl cells which are primarily 2 C stomata cells $[28,29,41,58]$. Other 2 C cells can eventually divide much later during secondary growth although the majority of cells in the central cylinder stay at $2 \mathrm{C} / 4 \mathrm{C}[28,41]$, observed here in the non-GFP populations (see Figure 1E,G). This means most of the $2 \mathrm{C}$ cells we sorted from the hypocotyl epidermis were primarily already fated to be stomatal cells. We can conclude that if there are transcriptional changes due to light conditions (which we did not test), we have to predict at the moment that they must be independent of large changes in the chromatin landscape, since the $24 \mathrm{~h}$ light treatment did not have a strong effect on the open chromatin pattern in this cell type.

Our experimental setup was designed to capture the largest amount of exponential growth in the hypocotyl, which occurs from day three to day four in both light- and dark-grown seedlings [28]. Hypocotyl growth is overwhelmingly accomplished by cell elongation via promoting endoreduplication in the dark and repressing it in the light, mediated by various light receptors [28,59]. Thus, part of the strong response to light should include a halting of endoreduplication, and in fact we observed this in our data as well. Cells that enter the endocycle program cannot resume mitotic divisions [60]. Cell growth as such is independent of cell divisions or cell wall synthesis and therefore requires expansins [2]. Two genes are likely involved in cell wall synthesis/signaling: ARABINOGALACTAN PROTEIN 12 (AT3G13520) and L-GALL RESPONSIVE GENE 1 (AT1G80240) have DARs (Table S1), suggestive of a halt to cell expansion, as we believe that DAR genes are likely downregulated in the light. While most of the genes we captured are not classically involved in endoreduplication [35], two other genes, AT5G15960 and AT5G45870, are involved in ABA signaling and have LARs. This is consistent with $\mathrm{ABA}$ as a negative regulator of endoreduplication $[2,30]$, since we presume that both genes were upregulated in our experiment.

Endoreduplication leads not only to larger cells, but also larger nuclei and more chromatin [61], and more complex nuclear morphology [37]. Constrained chromatin mobility increases with higher endopolyploid levels [62]. Our 16C nuclei adopted a more circular shape after one day of light exposure (Figure S7), implying some changes in genome packing with a halt to endoreduplication. LARs genes FANCONI ANEMIA COMPLEMENTATION GROUP M (AT1G35530), a highly conserved helicase and $A T 4 G 17240$, encoding a structural maintenance of chromosomes protein might be part of 
this process. Two other genes, HOMOLOG OF YEAST ADA2 2B (AT4G16420), which can stimulate acetyltransferase GCN5 on free histones or nucleosomes [63] and ARABIDOPSIS TÓXICOS EN LEVADURA 18 (AT4G17245), encoding a RING/U-box protein, might also take part. How these genes would lead to changes in the overall nuclear morphology is unclear at this point. Signaling through the hypocotyl between the shoot and root apical meristems occurs through the trafficking of mobile signals, which may be RNA or protein products [64], and five genes with LARs and three genes with DARs encode RNAs that cell-to-cell mobile [48] (Table S3). However, the four LAR genes that encode components of the photosynthesis machinery are unlikely to be directly connected to endoreduplication.

We speculate that we have likely captured genes involved in halting the endocycle process, as it would have proceeded to $32 \mathrm{C}$ if left in the dark [65]. If we presume that the already differentiated cells cannot de-differentiate as has been suggested before $[28,41]$, then it is likely those cells at the $8 \mathrm{C}$ level, or those just entering the $16 \mathrm{C}$ endocycle, were halted as they took on their new photomorphogenic fate. It is remarkable, then, that our experiment is able to capture such subtle changes in such a small cell pool. However, in order to get a more detailed overview about whether transcription activation triggers chromatin opening or histone remodeling attracts transcriptional activators in the hypocotyl, we need to perform further time course ATAC-seq and RNA-seq analyses, possibly also looking at the $4 \mathrm{C}$ and $8 \mathrm{C}$ nuclei. Using cortex or other cell-specific reporter lines would also be very interesting to explore, since it is conceivable that light-regulated transcriptional changes could also be occurring there. Deciphering such genome-wide information regarding photomorphogenesis and skotomorphogenesis will enhance our understanding of how the balance between these two antagonistic developmental processes is mediated to and through the chromatin environment.

\section{Materials and Methods}

\subsection{Plant Materials and Nuclei Sorting}

Arabidopsis used in this study were grown at $23{ }^{\circ} \mathrm{C}$ in long days $(16 \mathrm{~h}$ light $/ 8 \mathrm{~h}$ dark) on half-strength Murashige and Skoog (MS) medium [66] supplemented with $1 \%$ sucrose and $0.3 \%$ phytagel. The transgenic line used in this study was pAtML1:NLS:3xGFP in the Columbia-0 background [67]. For each replicate, 500 seeds were sterilized by washing with $70 \%$ ethanol for $20 \mathrm{~min}$ followed by extensive rinsing with water. For dark-treated samples, plants were grown on plates under the dark for 4 days. For light-treated samples, plants were grown in the dark for three days and transferred into the light for 1 day. Nuclei were isolated from fixed tissue and by flow cytometry as in [68] using a MoFlo (Beckman Coulter) with the following modifications: after initially gating nuclei from debris, each DAPI channel was compared by peak vs. integral to remove clumps and doublets, followed by inter-channel comparisons and monitoring the pulse-width of all channels to remove further clumps and debris. GFP was detected in the FL1 (530/34) via 488nm excitation. Pinhole spillover was accounted for by monitoring nuclei without GFP and sort regions adjusted appropriately. The extracted nuclei were stained with $0.5 \mu \mathrm{M}$ DAPI to reveal their ploidy levels; $2 \mathrm{C}$ and $16 \mathrm{C}$ nuclei were collected for downstream experiments. As we used a tissue-specific GFP marker, our nuclei consisted of epidermal cell nuclei after tissue dissection. Sorting was performed with Summit 5.2 (Beckman Coulter); re-rendering and basic statistic extraction of the data were done with FCS Express v.6 (deNovo); and graphing and statistical analysis were performed with JMP v.13 (SAS).

\subsection{ATAC-seq}

Our ATAC-seq experiment was performed with two biological replicates. For each replicate, 10,000 sorted $2 \mathrm{C}$ and $16 \mathrm{C}$ nuclei were collected in 1XPBS buffer and centrifuged at $3000 \times g$ at $4{ }^{\circ} \mathrm{C}$ for $5 \mathrm{~min}$. Then, $20 \mu \mathrm{L}$ Tn 5 transposase (Illumina) reactions were prepared according to [69] and nuclei were resuspended in this master mix. MinElute PCR Purification Kit (Qiagen) used to purify DNA. Selected Nextera index oligos (Illumina) were used for library preparation. 


\subsection{ATAC-seq Reads Processing and Peak Calling}

Our ATAC-seq data analysis was performed as previously published with minor differences [44]. Bowtie 2 v2.2.4 was used to align ATAC-seq reads to the Arabidopsis thaliana reference genome (TAIR10) [70]. ATAC-seq peaks were identified by using MACS 2 with the following parameters: "nomodel-shift -50-extsize 100-keep-dup $=1$ " [71]. A list of potentially accessible regions was created by merging the MACS2 identified peaks from all samples and number of raw reads mapped to each region for each condition was quantified using the multicov function in BedTools [72]. This count table was used to identify differentially accessible regions with the DESeq2 package in R [73]. The criteria to achieve differential peaks were $\log 2$ fold change greater than 1.5 to call gain-of-accessibility (UP) and loss-of-accessibility (DOWN) peaks and padj smaller than 0.01 . For GO analysis, differentially accessible genes from Deseq2 were uploaded to the plant GSEA with default parameters (Table S2) [74]. The ATAC-seq peaks called from each treatment and replicate, the peak count table, and the differentially accessible peaks can be found in Table S1. For the motif shown in Figure 3B, 160 bp regions from both sides of the midpoint of the differentially accessible regions are submitted to the MEME tool with the following parameters: "meme sequences.fa-dna-oc. -nostatus-time 18,000-mod zoops-nmotifs 4-minw 6-maxw 15-objfun classic-revcomp-markov_order 0" [49].

\subsection{Analysis of Gene Regulation in Microarray Datasets}

Affymetrix 25k files from AtGenExpress: “Light treatments” TAIR-ME00345 (www.arabidopsis. org) were imported into GeneSpring GX and GC-RMA normalized using the onboard "GC RMA File Processor" algorithm. Thereafter, the total dataset was normalized "specific samples per gene" to the dark control. Genes were inspected for two-fold changes over the dark control due to white light samples cross-comparing all time points (Table S4). TAIR9 was used as the genome reference.

Supplementary Materials: The following are available online at http://www.mdpi.com/2223-7747/9/11/1478/s1, Figure S1. ATAC-seq reads obtained from nuclei fixed with $4 \%$ and 1\% FA. Figure S2. Chromatin accessibility at PIFs and HY5 binding sites in different samples. Figure S3. Local chromatin accessibility around PIF genes. Figure S4. Klepikova Atlas expression of AT3G44970. Figure S5. MEME motif analysis on LARs. Figure S6. Chromatin accessibility around the upregulated genes upon 6-h light exposure from Sun et al. 2016 [55]. Figure S7. Nuclei circularity of dark and light treated GFP-positive epidermal hypocotyl 16C nuclei. Table S1: ATAC-seq peaks and gene assignments. Table S2: GO analysis of DAR and LAR gene lists. Table S3: Identification of genes that produce mobile gene products. Table S4: DAR and LAR genes in the AtGenExpress Light Experiments. Table S5: Overlap of DAR and LAR genes with the fifteen subgroups in Sun et al. 2016 [55].

Author Contributions: C.L. and K.W.B. conceived and designed the experiments. E.S.K. and N.F. prepared the nuclei for sorting. K.W.B. performed nucleus sorting. E.S.K. performed ATAC-seq experiments and analysis. E.S.K. and K.W.B. wrote the manuscript with contributions from all authors. All authors have read and agreed to the published version of the manuscript.

Funding: This work was supported by the European Research Council (ERC) under the European Union's Research and Innovation programme Horizon 2020 (grant agreement No. 757600).

Acknowledgments: We acknowledge computing support by the High Performance and Cloud Computing Group at the Zentrum für Datenverarbeitung of the University of Tübingen, the state of Baden-Württemberg through bwHPC and the German Research Foundation (DFG) through grant no. INST 37/935-1 FUGG.

Conflicts of Interest: The authors declare no conflict of interest.

Data Availability: Short read data are publicly available at the NCBI Sequence Read Archive as BioProject accession number PRJNA664913.

\section{References}

1. Von Arnim, A.; Deng, X.-W. LIGHT CONTROL OF SEEDLING DEVELOPMENT. Annu. Rev. Plant Physiol. Plant Mol. Biol. 1996, 47, 215-243. [CrossRef]

2. Gommers, C.M.M.; Monte, E. Seedling Establishment: A Dimmer Switch-Regulated Process between Dark and Light Signaling. Plant Physiol. 2018, 176, 1061-1074. [CrossRef] 
3. Chenge-Espinosa, M.; Cordoba, E.; Romero-Guido, C.; Toledo-Ortiz, G.; León, P. Shedding light on the methylerythritol phosphate (MEP)-pathway: Long hypocotyl 5 (HY5)/phytochrome-interacting factors (PIFs) transcription factors modulating key limiting steps. Plant J. 2018, 96, 828-841. [CrossRef] [PubMed]

4. Leivar, P.; Monte, E. PIFs: Systems integrators in plant development. Plant Cell 2014, 26, 56-78. [CrossRef] [PubMed]

5. Leivar, P.; Quail, P.H. PIFs: Pivotal components in a cellular signaling hub. Trends Plant Sci. 2011, 16, 19-28. [CrossRef]

6. De Lucas, M.; de Lucas, M.; Davière, J.-M.; Rodríguez-Falcón, M.; Pontin, M.; Iglesias-Pedraz, J.M.; Lorrain, S.; Fankhauser, C.; Blázquez, M.A.; Titarenko, E.; et al. A molecular framework for light and gibberellin control of cell elongation. Nature 2008, 451, 480-484. [CrossRef]

7. Feng, S.; Martinez, C.; Gusmaroli, G.; Wang, Y.; Zhou, J.; Wang, F.; Chen, L.; Yu, L.; Iglesias-Pedraz, J.M.; Kircher, S.; et al. Coordinated regulation of Arabidopsis thaliana development by light and gibberellins. Nature 2008, 451, 475-479. [CrossRef] [PubMed]

8. Leivar, P.; Monte, E.; Oka, Y.; Liu, T.; Carle, C.; Castillon, A.; Huq, E.; Quail, P.H. Multiple phytochrome-interacting bHLH transcription factors repress premature seedling photomorphogenesis in darkness. Curr. Biol. 2008, 18, 1815-1823. [CrossRef]

9. Leivar, P.; Tepperman, J.M.; Cohn, M.M.; Monte, E.; Al-Sady, B.; Erickson, E.; Quail, P.H. Dynamic Antagonism between Phytochromes and PIF Family Basic Helix-Loop-Helix Factors Induces Selective Reciprocal Responses to Light and Shade in a Rapidly Responsive Transcriptional Network in Arabidopsis. Plant Cell 2012, 24, 1398-1419. [CrossRef] [PubMed]

10. Shin, J.; Kim, K.; Kang, H.; Zulfugarov, I.S.; Bae, G.; Lee, C.-H.; Lee, D.; Choi, G. Phytochromes promote seedling light responses by inhibiting four negatively-acting phytochrome-interacting factors. Proc. Natl. Acad. Sci. USA 2009, 106, 7660-7665. [CrossRef]

11. Al-Sady, B.; Ni, W.; Kircher, S.; Schäfer, E.; Quail, P.H. Photoactivated phytochrome induces rapid PIF3 phosphorylation prior to proteasome-mediated degradation. Mol. Cell 2006, 23, 439-446. [CrossRef] [PubMed]

12. Shen, Y.; Khanna, R.; Carle, C.M.; Quail, P.H. Phytochrome induces rapid PIF5 phosphorylation and degradation in response to red-light activation. Plant Physiol. 2007, 145, 1043-1051. [CrossRef]

13. Shen, H.; Zhu, L.; Castillon, A.; Majee, M.; Downie, B.; Huq, E. Light-Induced Phosphorylation and Degradation of the Negative Regulator PHYTOCHROME-INTERACTING FACTOR1 from Arabidopsis Depend upon Its Direct Physical Interactions with Photoactivated Phytochromes. Plant Cell 2008, 20, 1586-1602. [CrossRef]

14. Oyama, T.; Shimura, Y.; Okada, K. The Arabidopsis HY5 gene encodes a bZIP protein that regulates stimulus-induced development of root and hypocotyl. Genes Dev. 1997, 11, 2983-2995. [CrossRef] [PubMed]

15. Zhang, H.; He, H.; Wang, X.; Wang, X.; Yang, X.; Li, L.; Deng, X.W. Genome-wide mapping of the HY5-mediated gene networks in Arabidopsis that involve both transcriptional and post-transcriptional regulation. Plant J. 2011, 65, 346-358. [CrossRef]

16. Lee, J.; He, K.; Stolc, V.; Lee, H.; Figueroa, P.; Gao, Y.; Tongprasit, W.; Zhao, H.; Lee, I.; Deng, X.W. Analysis of transcription factor HY5 genomic binding sites revealed its hierarchical role in light regulation of development. Plant Cell 2007, 19, 731-749. [CrossRef] [PubMed]

17. Kurihara, Y.; Makita, Y.; Kawashima, M.; Hamasaki, H.; Yamamoto, Y.Y.; Matsui, M. Next-generation sequencing of genomic DNA fragments bound to a transcription factor in vitro reveals its regulatory potential. Genes 2014, 5, 1115-1131. [CrossRef]

18. Zhao, L.; Peng, T.; Chen, C.-Y.; Ji, R.; Gu, D.; Li, T.; Zhang, D.; Tu, Y.-T.; Wu, K.; Liu, X. HY5 interacts with the histone deacetylase HDA15 to repress hypocotyl cell elongation in photomorphogenesis. Plant Physiol. 2019, 180, 1450-1466. [CrossRef]

19. Perrella, G.; Zioutopoulou, A.; Headland, L.R.; Kaiserli, E. The impact of light and temperature on chromatin organization and plant adaptation. J. Exp. Bot. 2020, 71, 5247-5255. [CrossRef]

20. Jang, I.-C.; Chung, P.J.; Hemmes, H.; Jung, C.; Chua, N.-H. Rapid and reversible light-mediated chromatin modifications of Arabidopsis phytochrome A locus. Plant Cell 2011, 23, 459-470. [CrossRef]

21. Bourbousse, C.; Barneche, F.; Laloi, C. Plant Chromatin Catches the Sun. Front. Plant Sci. 2019, 10, 1728. [CrossRef]

22. Liu, C.; Weigel, D. Chromatin in 3D: Progress and prospects for plants. Genome Biol. 2015, 16, 170. [CrossRef] 
23. Del Pozo, J.C.; Ramirez-Parra, E. Whole genome duplications in plants: An overview from Arabidopsis. J. Exp. Bot. 2015, 66, 6991-7003. [CrossRef] [PubMed]

24. Galbraith, D.W.; Harkins, K.R.; Knapp, S. Systemic Endopolyploidy in Arabidopsis thaliana. Plant Physiol. 1991, 96, 985-989. [CrossRef]

25. Fox, D.T.; Duronio, R.J. Endoreplication and polyploidy: Insights into development and disease. Development 2013, 140, 3-12. [CrossRef]

26. Mendell, J.E.; Clements, K.D.; Choat, J.H.; Angert, E.R. Extreme polyploidy in a large bacterium. Proc. Natl. Acad. Sci. USA 2008, 105, 6730-6734. [CrossRef]

27. Katagiri, Y.; Hasegawa, J.; Fujikura, U.; Hoshino, R.; Matsunaga, S.; Tsukaya, H. The coordination of ploidy and cell size differs between cell layers in leaves. Development 2016, 143, 1120-1125. [CrossRef] [PubMed]

28. Gendreau, E.; Traas, J.; Desnos, T.; Grandjean, O.; Caboche, M.; Höfte, H. Cellular basis of hypocotyl growth in Arabidopsis thaliana. Plant Physiol. 1997, 114, 295-305. [CrossRef]

29. Saibo, N.J.M.; Vriezen, W.H.; Beemster, G.T.S.; Van Der Straeten, D. Growth and stomata development of Arabidopsis hypocotyls are controlled by gibberellins and modulated by ethylene and auxins. Plant J. 2003, 33, 989-1000. [CrossRef]

30. Lorrai, R.; Boccaccini, A.; Ruta, V.; Possenti, M.; Costantino, P.; Vittorioso, P. Abscisic acid inhibits hypocotyl elongation acting on gibberellins, DELLA proteins and auxin. AoB Plants 2018, 10, ly061.

31. Tsukaya, H. Does ploidy level directly control cell size? Counterevidence from Arabidopsis genetics. PLOS ONE 2013, 8, e83729. [CrossRef]

32. Nagl, W. DNA endoreduplication and polyteny understood as evolutionary strategies. Nature 1976, 261, 614-615. [CrossRef]

33. Baluška, F.; Kubica, Š. Relationships Between the Content of Basic Nuclear Proteins, Chromatin Structure, rDNA Transcription and Cell Size in Different Tissues7. J. Exp. Bot. 1992, 43, 991-996. [CrossRef]

34. Kondorosi, E.; Roudier, F.; Gendreau, E. Plant cell-size control: Growing by ploidy? Curr. Opin. Plant Biol. 2000, 3, 488-492. [CrossRef]

35. Sugimoto-Shirasu, K.; Roberts, K. "Big it up": Endoreduplication and cell-size control in plants. Curr. Opin. Plant Biol. 2003, 6, 544-553. [CrossRef] [PubMed]

36. Marguerat, S.; Bähler, J. Coordinating genome expression with cell size. Trends Genet. 2012, 28, 560-565. [CrossRef]

37. Bourdon, M.; Pirrello, J.; Cheniclet, C.; Coriton, O.; Bourge, M.; Brown, S.; Moïse, A.; Peypelut, M.; Rouyère, V.; Renaudin, J.-P.; et al. Evidence for karyoplasmic homeostasis during endoreduplication and a ploidy-dependent increase in gene transcription during tomato fruit growth. Development 2012, 139, 3817-3826. [CrossRef]

38. Galbraith, D.W.; Harkins, K.R.; Maddox, J.M.; Ayres, N.M.; Sharma, D.P.; Firoozabady, E. Rapid flow cytometric analysis of the cell cycle in intact plant tissues. Science 1983, 220, 1049-1051. [CrossRef]

39. Zhang, C.; Gong, F.C.; Lambert, G.M.; Galbraith, D.W. Cell type-specific characterization of nuclear DNA contents within complex tissues and organs. Plant Methods 2005, 1, 7. [CrossRef]

40. Zhang, C.; Barthelson, R.A.; Lambert, G.M.; Galbraith, D.W. Global characterization of cell-specific gene expression through fluorescence-activated sorting of nuclei. Plant Physiol. 2008, 147, 30-40. [CrossRef]

41. Gendreau, E.; Höfte, H.; Grandjean, O.; Brown, S.; Traas, J. Phytochrome controls the number of endoreduplication cycles in the Arabidopsis thaliana hypocotyl. Plant J. 1998, 13, 221-230. [CrossRef] [PubMed]

42. Lu, Z.; Hofmeister, B.T.; Vollmers, C.; DuBois, R.M.; Schmitz, R.J. Combining ATAC-seq with nuclei sorting for discovery of cis-regulatory regions in plant genomes. Nucleic Acids Res. 2017, 45, e41. [CrossRef] [PubMed]

43. Marion-Poll, L.; Montalban, E.; Munier, A.; Hervé, D.; Girault, J.-A. Fluorescence-activated sorting of fixed nuclei: A general method for studying nuclei from specific cell populations that preserves post-translational modifications. Eur. J. Neurosci. 2014, 39, 1234-1244. [CrossRef]

44. Hu, B.; Wang, N.; Bi, X.; Karaaslan, E.S.; Weber, A.-L.; Zhu, W.; Berendzen, K.W.; Liu, C. Plant lamin-like proteins mediate chromatin tethering at the nuclear periphery. Genome Biol. 2019, 20, 87. [CrossRef]

45. Pfeiffer, A.; Shi, H.; Tepperman, J.M.; Zhang, Y.; Quail, P.H. Combinatorial complexity in a transcriptionally centered signaling hub in Arabidopsis. Mol. Plant 2014, 7, 1598-1618. [CrossRef] 
46. Burko, Y.; Seluzicki, A.; Zander, M.; Pedmale, U.V.; Ecker, J.R.; Chory, J. Chimeric Activators and Repressors Define HY5 Activity and Reveal a Light-Regulated Feedback Mechanism. Plant Cell 2020, 32, 967-983. [CrossRef]

47. Pham, V.N.; Kathare, P.K.; Huq, E. Phytochromes and Phytochrome Interacting Factors. Plant Physiol. 2018, 176, 1025-1038. [CrossRef] [PubMed]

48. Thieme, C.J.; Rojas-Triana, M.; Stecyk, E.; Schudoma, C.; Zhang, W.; Yang, L.; Miñambres, M.; Walther, D.; Schulze, W.X.; Paz-Ares, J.; et al. Endogenous Arabidopsis messenger RNAs transported to distant tissues. Nat. Plants 2015, 1, 15025. [CrossRef]

49. Bailey, T.L.; Elkan, C. Fitting a mixture model by expectation maximization to discover motifs in bipolymers. Proc. Int. Conf. Intell. Syst. Mol. Biol. 1994, 2, 28-36.

50. Sakuraba, Y.; Jeong, J.; Kang, M.-Y.; Kim, J.; Paek, N.-C.; Choi, G. Phytochrome-interacting transcription factors PIF4 and PIF5 induce leaf senescence in Arabidopsis. Nat. Commun. 2014, 5, 4636. [CrossRef]

51. Song, Y.H.; Yoo, C.M.; Hong, A.P.; Kim, S.H.; Jeong, H.J.; Shin, S.Y.; Kim, H.J.; Yun, D.-J.; Lim, C.O.; Bahk, J.D.; et al. DNA-binding study identifies C-box and hybrid C/G-box or C/A-box motifs as high-affinity binding sites for STF1 and LONG HYPOCOTYL5 proteins. Plant Physiol. 2008, 146, 1862-1877. [CrossRef]

52. Hobo, T.; Asada, M.; Kowyama, Y.; Hattori, T. ACGT-containing abscisic acid response element (ABRE) and coupling element 3 (CE3) are functionally equivalent. Plant J. 1999, 19, 679-689. [CrossRef] [PubMed]

53. Sibéril, Y.; Doireau, P.; Gantet, P. Plant bZIP G-box binding factors. Eur. J. Biochem. 2001, 268, 5655-5666. [CrossRef] [PubMed]

54. Gómez-Porras, J.L.; Riaño-Pachón, D.M.; Dreyer, I.; Mayer, J.E.; Mueller-Roeber, B. Genome-wide analysis of ABA-responsive elements ABRE and CE3 reveals divergent patterns in Arabidopsis and rice. BMC Genom. 2007, 8, 260. [CrossRef]

55. Sun, N.; Wang, J.; Gao, Z.; Dong, J.; He, H.; Terzaghi, W.; Wei, N.; Deng, X.W.; Chen, H. Arabidopsis SAURs are critical for differential light regulation of the development of various organs. Proc. Natl. Acad. Sci. USA 2016, 113, 6071-6076. [CrossRef]

56. Yu, Y.; Huang, R. Integration of Ethylene and Light Signaling Affects Hypocotyl Growth in Arabidopsis. Front. Plant Sci. 2017, 8, 57. [CrossRef]

57. Koornneef, M.; Rolff, E.; Spruit, C.J.P. Genetic Control of Light-inhibited Hypocotyl Elongation in Arabidopsis thaliana (L.) Heynh. Z. Pflanzenphysiol. 1980, 100, 147-160. [CrossRef]

58. Dan, H.; Imaseki, H.; Wasteneys, G.O.; Kazama, H. Ethylene stimulates endoreduplication but inhibits cytokinesis in cucumber hypocotyl epidermis. Plant Physiol. 2003, 133, 1726-1731. [CrossRef] [PubMed]

59. Tsumoto, Y.; Yoshizumi, T.; Kuroda, H.; Kawashima, M.; Ichikawa, T.; Nakazawa, M.; Yamamoto, N.; Matsui, M. Light-dependent polyploidy control by a CUE protein variant in Arabidopsis. Plant Mol. Biol. 2006, 61, 817-828. [CrossRef]

60. Roeder, A.H.K.; Chickarmane, V.; Cunha, A.; Obara, B.; Manjunath, B.S.; Meyerowitz, E.M. Variability in the control of cell division underlies sepal epidermal patterning in Arabidopsis thaliana. PLoS Biol. 2010, 8 , e1000367. [CrossRef]

61. Robinson, D.O.; Coate, J.E.; Singh, A.; Hong, L.; Bush, M.; Doyle, J.J.; Roeder, A.H.K. Ploidy and Size at Multiple Scales in the Arabidopsis Sepal. Plant Cell 2018, 30, 2308-2329. [CrossRef] [PubMed]

62. Kato, N.; Lam, E. Chromatin of endoreduplicated pavement cells has greater range of movement than that of diploid guard cells in Arabidopsis thaliana. J. Cell Sci. 2003, 116, 2195-2201. [CrossRef]

63. Lai, J.; Jiang, J.; Wu, Q.; Mao, N.; Han, D.; Hu, H.; Yang, C. The Transcriptional Coactivator ADA2b Recruits a Structural Maintenance Protein to Double-Strand Breaks during DNA Repair in Plants. Plant Physiol. 2018, 176, 2613-2622. [CrossRef]

64. Jackson, M.D.; Xu, H.; Duran-Nebreda, S.; Stamm, P.; Bassel, G.W. Topological analysis of multicellular complexity in the plant hypocotyl. eLife 2017, 6. [CrossRef]

65. Yoshizumi, T.; Tsumoto, Y.; Takiguchi, T.; Nagata, N.; Yamamoto, Y.Y.; Kawashima, M.; Ichikawa, T.; Nakazawa, M.; Yamamoto, N.; Matsui, M. Increased level of polyploidy1, a conserved repressor of CYCLINA2 transcription, controls endoreduplication in Arabidopsis. Plant Cell 2006, 18, 2452-2468. [CrossRef]

66. Murashige, T.; Skoog, F. A revised medium for rapid growth and bio assays with tobacco tissue cultures. Physiol. Plant. 1962, 15, 473-497. [CrossRef]

67. Takada, S.; Jürgens, G. Transcriptional regulation of epidermal cell fate in the Arabidopsis embryo. Development 2007, 134, 1141-1150. [CrossRef] [PubMed] 
68. Zhu, W.; Hu, B.; Becker, C.; Doğan, E.S.; Berendzen, K.W.; Weigel, D.; Liu, C. Altered chromatin compaction and histone methylation drive non-additive gene expression in an interspecific Arabidopsis hybrid. Genome Biol. 2017, 18, 157. [CrossRef]

69. Bajic, M.; Maher, K.A.; Deal, R.B. Identification of Open Chromatin Regions in Plant Genomes Using ATAC-Seq. Methods Mol. Biol. 2018, 1675, 183-201.

70. Langmead, B.; Salzberg, S.L. Fast gapped-read alignment with Bowtie 2. Nat. Methods 2012, 9, 357-359. [CrossRef]

71. Liu, T. Use Model-Based Analysis of ChIP-Seq (MACS) to Analyze Short Reads Generated by Sequencing Protein-DNA Interactions in Embryonic Stem Cells. In Stem Cell Transcriptional Networks: Methods and Protocols; Kidder, B.L., Ed.; Springer: New York, NY, USA, 2014; pp. 81-95. ISBN 9781493905126.

72. Quinlan, A.R.; Hall, I.M. BEDTools: A flexible suite of utilities for comparing genomic features. Bioinformatics 2010, 26, 841-842. [CrossRef]

73. Love, M.I.; Huber, W.; Anders, S. Moderated estimation of fold change and dispersion for RNA-seq data with DESeq2. Genome Biol. 2014, 15, 550. [CrossRef]

74. Yi, X.; Du, Z.; Su, Z. PlantGSEA: A gene set enrichment analysis toolkit for plant community. Nucleic Acids Res. 2013, 41, W98-W103. [CrossRef]

Publisher's Note: MDPI stays neutral with regard to jurisdictional claims in published maps and institutional affiliations.

(C) 2020 by the authors. Licensee MDPI, Basel, Switzerland. This article is an open access article distributed under the terms and conditions of the Creative Commons Attribution (CC BY) license (http://creativecommons.org/licenses/by/4.0/). 